\title{
Prevalência e relação de fatores emocionais e clínicos em pacientes com discopatia degenerativa
}

\author{
Prevalence and relationship of emotional and clinical factors in \\ patients with degenerative disc disease \\ La prevalencia y la relación de los factores clínicos y los trastornos \\ emocionales en pacientes con discopatia degenerativa del disco
}

\author{
Vivian Amaral' \\ Luis Marchi ${ }^{2}$ \\ Leonardo Oliveira ${ }^{3}$ \\ Luiz Pimenta ${ }^{4}$
}

\section{RESUMO}

Objetivos: descrever as condições psicossociais, no momento da indicação cirúrgica, dos pacientes com dor lombar crônica causada por discopatia degenerativa. Métodos: em uma série de 32 casos de discopatia degenerativa eletivos para cirurgia, foi realizada avaliação psicológica pré-operatória. Nesta avaliação, foram colhidos dados demográficos e sociais: sexo, idade, situação ocupacional atual, ocorrência de afastamento por invalidez; e dados clínico-psicológicos: dor, restrição física, utilização de medicamento psiquiátrico controlado; grau de ansiedade; grau de depressão; grau de desesperança; expectativas frente ao objetivo cirúrgico. Resultados: o grupo foi composto de 13 mulheres e 14 homens, com média de idade de 44,3 anos (23 a 65 anos). Os parâmetros de dor e restrição física estão dentro de valores geralmente indicativos de cirurgia, não indicando nenhuma peculiaridade no grupo

\section{ABSTRACT}

objectives: to describe the psychosocial conditions, at the time of surgical indication, of patients with chronic low back pain caused by degenerative disc disease. Methods: in a series of 32 cases of degenerative disc disease elective surgery, a psychological evaluation was performed before the surgery. In this evaluation, socio-demographic data were collected: gender; age; present working status; occurrence of withdrawal due to disability; and clinical-psychological data: pain; physical restraint; use of psychiatric medication controlled; degree of anxiety; degree of depression; degree of hopelessness; expectations before the surgical goal. Results: the group was composed of 13 women and 14 men, mean age of 44.3 years old (23 to 65 years). The parameters of pain and physical restraint were compared to cases usually indicative of surgery, indicating no

\section{RESUMEN}

Objetivos: describir las condiciones psicosociales, en el momento de la indicación quirúrgica, de pacientes con dolor lumbar crónico causado por discopatia degenerativa del disco. Métodos: en una serie de 32 casos de discopatia degenerativa del disco, se llevó a cabo una evaluación psicológica antes de la cirugía para la cirugía electiva. En esta evaluación, se recogieron datos socio-demográficos: de género; edad; situación laboral actual; la aparición de retiro debido a la discapacidad; y la clínica del dolor psicológico: la restricción fisica; uso de medicación psiquiátrica controlada; el grado de ansiedad; el grado de depresión; el grado de la desesperanza; expectativas antes de la meta quirúrgica. Resultados: el grupo estaba compuesto por 13 mujeres y 14 hombres, edad media de 44,3 años (23 a 65 años). Los parámetros de dolor y de la restricción fisica están en la acción suele ser indicativa de la cirugía, lo que indica ninguna peculiaridad en

\footnotetext{
Instituto de Patologia da Coluna - IPC - São Paulo (SP), Brasil.

1 Psicóloga do Instituto de Patologia da Coluna - IPC - São Paulo (SP), Brasil; Psicóloga da Irmandade da Santa Casa de Misericórdia de São Paulo - ISCMSP _ São Paulo (SP), Brasil.

2 Mestre; Biomédico; Pesquisador do Instituto da Patologia de Coluna - IPC - São Paulo (SP), Brasil.

${ }^{3}$ Biomédico; Pesquisador Coordenador do Instituto de Patologia da Coluna - IPC - São Paulo (SP), Brasil; Pós-graduando (Mestrado) do Departamento de Diagnóstico por Imagem da Universidade Federal de São Paulo - UNIFESP - São Paulo (SP), Brasil.

${ }^{4}$ Doutor; Diretor Médico do Instituto de Patologia da Coluna - IPC - São Paulo (SP), Brasil; Professor da Universidade de San Diego - UCSD - San Diego (CA),

Estados Unidos.
} 
estudado. Foi evidenciada uma alta e correlacionada prevalência de alterações psicológicas nos pacientes: $50 \%$ em quadro depressivo, $54 \%$ em quadro de ansiedade e $22 \%$ em quadro de desesperança. Além disso, foi possível evidenciar altos índices de afastamento do trabalho por invalidez física e correlação da presença de ansiedade e/ou depressão com casos em que haviam expectativas deturpadas quanto ao resultado cirúrgico. Conclusões: dor lombar crônica é acompanhada de quadros de dor e de alterações psicológicas, como ansiedade, depressão e desesperança. Esses parâmetros evidenciam a necessidade de avaliação psicológica pré-cirúrgica e acompanhamento posterior, visando à reabilitação do paciente.

DESCRITORES: Psicologia médica; Doença crônica; Coluna vertebral; Depressão; Ansiedade; Dor lombar peculiarity in the studied group. We demonstrated a high and correlated prevalence of psychological changes in patients: $50 \%$ of depression, $54 \%$ of anxiety and $22 \%$ of hopelessness. Furthermore, it was possible to show high rates of absence from work due to physical disability and relation to the presence of anxiety and/or depression with cases in which expectations were misleading according to the surgical objective. Conclusions: chronic low back pain is accompanied by clinical pictures of pain and psychological disorders such as anxiety, depression and hopelessness. These parameters indicate the need for pre-surgical psychological evaluation and posterior follow-up, aiming at the rehabilitation of the patient.

KEYWORDS: Psychology, medical; Chronic disease; Spine; Depression; Anxiety; Low back pain el grupo de estudio. Hemos demostrado una alta prevalencia y las correlaciones de cambios psicológicos en pacientes: el $50 \%$ en la depresión, el $54 \%$ en el marco de la ansiedad y el $22 \%$ en el marco de la desesperanza. Además, era posible mostrar altas tasas de ausencia del trabajo debido a la discapacidad y la correlación de la presencia fisica de la ansiedad y la depresión de los casos donde las expectativas eran engañosas en cuanto a los resultados. Conclusiones: el dolor lumbar crónico se acompaña de imágenes de dolor y los trastornos psicológicos tales como ansiedad, depresión y la desesperanza. Estos parámetros indican la necesidad de una evaluación psicológica antes de la cirugía y el seguimiento, con miras a la rehabilitación del paciente.

DESCRITORES: Psicología médica; Enfermedad crónica; Columna vertebral; Depresión; Ansiedad; Dolor de la región lumbar

\section{INTRODUÇÃO}

Já é bem conhecido, hoje em dia, que cerca de $80 \%$ da população mundial terá dor nas costas em algum momento da vida ${ }^{1}$. Quase $99 \%$ destes pacientes conseguem reverter o quadro álgico com utilização de analgésicos ou tratamentos físicos conservadores ${ }^{2}$, por meio das diversas modalidades de correção postural ou de reforço da musculatura paravertebral. Porém, o restante não se beneficia de práticas não-cirúrgicas, de modo que as dores sentidas perduram e se tornam problemas crônicos.

Nas síndromes de dor crônica, como dores de coluna e fribromialgia, existe alta prevalência de alterações emocionais importantes, como quadros de depressão e ansiedade $^{3,4}$. Pessoas que sofrem de dor crônica de coluna podem acabar desenvolvendo sintomas depressivos, uma vez que sentem dores constantes e incapacitantes que podem, em alguns momentos, impedi-los de exercerem suas atividades profissionais, pessoais e sociais, e isso pode fazer com que as limitações físicas acarretem em prejuízos emocionais. Tendo em vista esse preceito, a prevalência destes problemas emocionais foi comprovadamente maior em pessoas com diagnóstico de dor discogênica do que pessoas saudáveis 5 .

A dor lombar crônica pode levar a uma diminuição da capacidade de participar de uma variedade de atividades como trabalho, lazer e interação com os familiares e amigos. Esta situação conduz a uma espiral descendente física e emocional, que tem sido chamado de "descondicionamento físico e mental"'.

Um fator fundamental para a reabilitação do paciente é a adesão ao tratamento pós-cirúrgico, como os cuidados iniciais e os progressivos exercícios físicos. Caso o paciente esteja mentalmente ou socialmente desmotivado, ele provavelmente não se sentirá impelido a realizar o programa de reabilitação, comprometendo os resultados esperados e realimentando o ciclo da doença.

A depressão ocupa o $4^{\circ}$ lugar na lista dos maiores problemas mundiais ${ }^{7}$ e, ainda, epidemiologistas alertam que até 2020 poderá ser a segunda causa de incapacitação para o trabalho ${ }^{8}$. Tendo em vista que pacientes com depressão apresentam quatro vezes mais dores restritivas do que pessoas sem alterações emocionais ${ }^{9}$, e que estas alterações se relacionam intimamente com os quadros álgicos, observase a necessidade de avaliação psicológica juntamente com a avaliação clínica.

Da mesma forma que os fatores emocionais, fatores sociais e financeiros influenciam na resolução de quadros de dor crônica, a compensação do trabalhador (em inglês, workers' compensation) e afastamentos do trabalho por invalidez podem ser vistos pelos pacientes como recompensas, e consequentemente o quadro crônico não é facilmente resolvido. Ainda, a sensibilidade à dor é aumentada em casos que envolvem incentivos fiscais e financeiros ${ }^{10}$. 
Sendo assim, o objetivo deste trabalho foi descrever e correlacionar a prevalência de fatores psicossociais em um grupo de pacientes encaminhados para cirurgia por diagnóstico de dor discogênica crônica.

\section{MÉTODOS}

Foi realizado um estudo descritivo prospectivo com 32 pacientes, todos diagnosticados com dor crônica por conta de discopatia degenerativa do disco intervertebral (DDD). Todos os indivíduos aqui apresentados foram pacientes após análise médica no Instituto de Patologia da Coluna (IPC, São Paulo, Brasil) e, por não terem respondido a tratamentos conservadores, foram indicados a procedimentos cirúrgicos.

Em visitas independentes e posteriores à indicação cirúrgica, um único psicólogo realizou durante entrevista a avaliação de diversos fatores psicossociais destes pacientes e realizou aplicação de testes psicométricos direcionados. Dentre os itens avaliados, estão: situação ocupacional, ocorrência de tratamento com medicamento psiquiátrico controlado, ocorrência de acompanhamento psicológico, abrangência da dor sentida, expectativas sobre o resultado cirúrgico, grau de depressão (via questionário "Beck Depression Inventory" $\mathrm{BDI})^{11,12}$, grau de ansiedade (via questionário "Beck Anxiety Inventory" - BAI) ${ }^{13}$, grau de desesperança (via questionário "Beck Hopelessness Scale" - BHS) $)^{14}$, grau de dor (via questionário "Visual Analogue Scale" - VAS) ${ }^{15}$, e grau de restrição física (via questionário "Oswestry" - ODI) ${ }^{16}$.

Para categorizar os dados numéricos obtidos no questionário $\mathrm{BDI}$, os intervalos de pontuações utilizados foram: resultados $\geq 0$ e $<11$ : ocorrência mínima; resultados $\geq 11$ e $<19$ : ocorrência leve; resultados $\geq 20$ e $<35$ : ocorrência moderada; resultados $\geq 35 \mathrm{e}<63$ : ocorrência grave.

Para categorizar os dados numéricos obtidos no questionário BAI, os intervalos de pontuações utilizados foram: resultados $\geq 0$ e $<10$ : ocorrência mínima; resultados $\geq 11$ e $<19$ : ocorrência leve; resultados $\geq 20$ e $<30$ : ocorrência moderada; resultados $\geq 31 \mathrm{e}<63$ : ocorrência grave.

Para categorizar os dados numéricos obtidos no questionário BHS, utilizaram-se os seguintes intervalos de pontuações: resultados $\geq 0$ e $<4$ : ocorrência mínima; resultados $\geq 4$ e $<8$ : ocorrência leve; resultados $\geq 9$ e $<13$ : ocorrência moderada; resultados $\geq 14$ e $<20$ : ocorrência grave.

Para análises estatísticas, foi utilizado o software Microsoft Office Excel $^{\mathbb{Q}}$, usando como ferramentas o teste $t$ de Student, considerando resultados com valores de $\mathrm{p} \leq 0,05$ estatisticamente significativos, e a regressão linear, considerando que valores de $\mathrm{R}^{2} \geq 0,4$ apontam que as variáveis estavam correlacionadas.

\section{RESULTADOS}

Primeiramente, foi possível observar que a amostra de pacientes utilizada foi homogeneamente distribuída entre os sexos (Tabela 1), o que evita qualquer interferência de gênero nos resultados globais aqui obtidos. A maior prevalência de DDD dentro do grupo está em L4L5, sendo ela unicamente neste nível ou se estendendo para L3L4 e/ou L5S1.
Ainda na tabela 1 , é possível observar um fenômeno alarmante: quase metade de todos pacientes do estudo estava em situação de afastamento do trabalho por motivo de invalidez física (temporária ou permanente). Além disso, da parcela com situação ocupacional ativa, $25 \%$ já foram afastados alguma vez (dados não mostrados).

por meio da quantificação das condições clínicas, dor (questionário VAS) e limitação física (questionário ODI) (Tabela 2), foi possível observar que a média foi alta, característica de pacientes com indicação a procedimentos cirúrgicos.

A partir de questionários de avaliação psicológica, foi revelado um panorama que chamou a atenção dos pesquisadores: a maior parte dos pacientes se encontrava em

\section{TABELA 1 - Dados demográficos da amostra}

\begin{tabular}{lc}
\hline Variável & Média DP ou porcentagem \\
\hline Gênero & \\
Masculino & $53,1 \%$ \\
Feminino & $46,9 \%$ \\
Idade & $44,5 \pm 9,5$ \\
Níveis afetados por DDD & \\
L2L3 & $6,3 \%$ \\
L3L4 & $12,5 \%$ \\
L3L4/L4L5/L5S1 & $3,1 \%$ \\
L4L5 & $34,4 \%$ \\
L4L5/L5S1 & $28,1 \%$ \\
L5S1 & $15,6 \%$ \\
Situação ocupacional & \\
Afastado & $43,8 \%$ \\
Ativo & $31,3 \%$ \\
Do lar & $9,4 \%$ \\
Desempregado & $6,3 \%$ \\
Aposentado & $9,4 \%$ \\
\hline
\end{tabular}

DP: desvio padrão; DDD: discopatia degenerativa do disco intervertebral.

\section{TABELA 2 - Dados psicoclínicos}

\begin{tabular}{lc}
\hline Variável & Média \pm DP ou porcentagem \\
\hline VAS & $75,5 \pm 23,6$ \\
ODI & $45,1 \pm 19,1$ \\
Depressão & $52 \%$ \\
Leve & $24 \%$ \\
Moderado & $28 \%$ \\
Ansiedade & $56 \%$ \\
Leve & $28 \%$ \\
Moderado & $28 \%$ \\
Desesperança & $21,4 \%$ \\
Leve & $10,7 \%$ \\
Moderado & $10,7 \%$ \\
Uso de medicação & \\
Psiquiátrica controlada & $48,4 \%$ \\
Expectativas incoerentes & $38,7 \%$ \\
\hline DP. desvio padrõo:VAS: Visual Analoge Scale; ODl $:$ questiońrio Oswestr.
\end{tabular}

DP: desvio padrão; VAS: Visual Analogue Scale; ODI: questionário Oswestry. 
quadros de ansiedade e/ou depressão. Constatou-se, ainda, que o quadro psicológico de desesperança estava presente em boa parcela do grupo estudado (Tabela 2). Por meio de análise cruzada entre as pontuações dos questionários de depressão, ansiedade e desesperança, foi possível mostrar que tais alterações emocionais estão correlacionadas nos pacientes (Figuras 1 a 3), ou seja, se o paciente tem um desses quadros psicológicos está predisposto a apresentar também os outros aqui estudados.

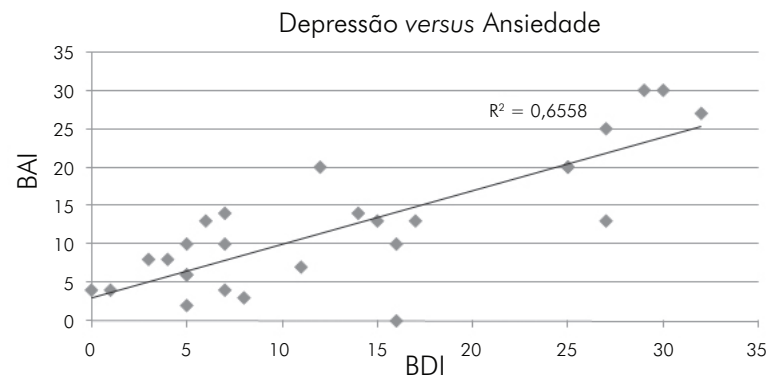

Figura 1

Relação de níveis de depressão e ansiedade. Cada ponto se refere às pontuações obtidas para cada sujeito pelos questionários BDI (Beck Depression Inventory) e BAI (Beck Anxiety Inventory). Por meio da aplicação de regressão linear, foi obtido o valor do $R^{2}=0,6558$.

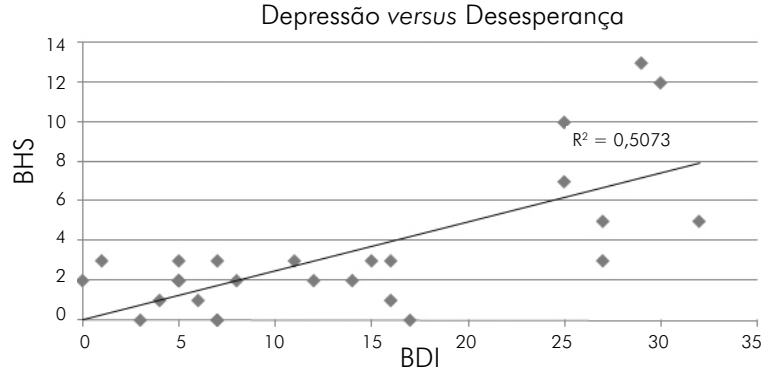

Figura 2

Relação de níveis de depressão e desesperança. Cada ponto se refere às pontuações obtidas para cada sujeito pelos questionários BDI (Beck Depression Inventory) e BHS (Beck Hopelessnes Scale). Por meio da aplicação de regressão linear, foi obtido o valor do $R^{2}=0,5073$.

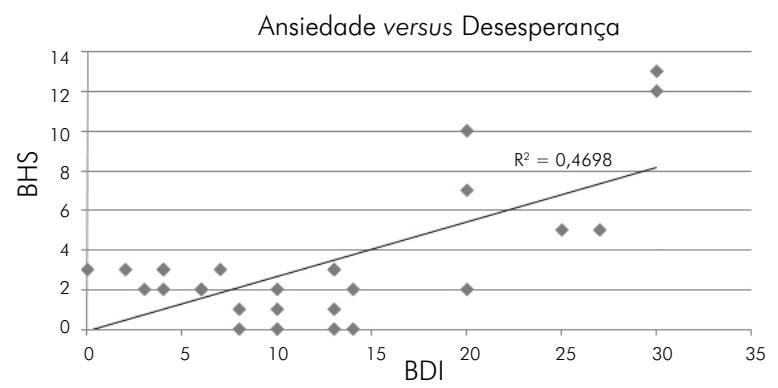

Figura 3

Relação de níveis de ansiedade e desesperança. Cada ponto se refere às pontuações obtidas para cada sujeito pelos questionários BAI (Beck Anxiety Inventory) e BHS (Beck Hopelessnes Scale). Por meio da aplicação de regressão linear, foi obtido o valor do $R^{2}=0,4698$.
Junto a esses dados de fatores psicológicos, verificouse que a ocorrência atual ou anterior de tratamento com medicamentos psiquiátrico controlados era de quase metade do grupo (Tabela 2).

Outro dado relevante encontrado após a avaliação psicológica foi que, apesar de ter passado em consultas clínicas explicativas do tratamento cirúrgico que seria adotado e os respectivos riscos/benefícios, a impactante porcentagem de $38,7 \%$ dos pacientes tinham expectativas deturpadas diante dos benefícios que eles poderiam obter (Tabela 2), sendo que quase a totalidade idealizava uma cura expansível e não limitada ao objetivo cirúrgico.

Com os dados de fatores psicológicos estratificados em gêneros, pôde-se observar uma clara diferença entre as prevalências em mulheres e homens, sendo que a

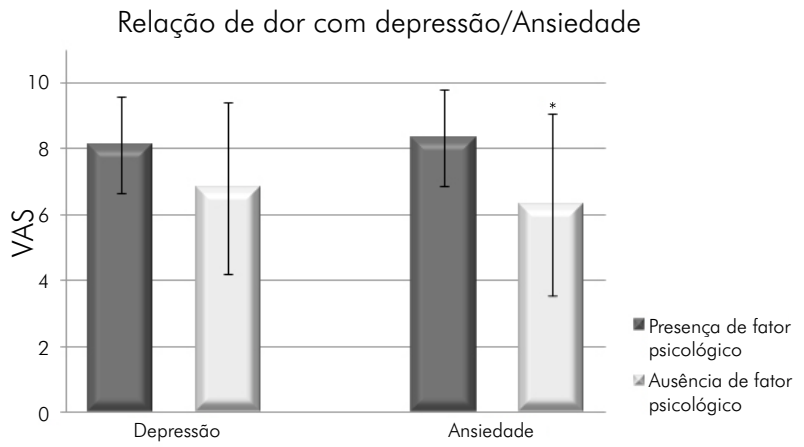

Figura 4

Comparação do grau de dor com depressão ou ansiedade. As pontuações do questionário sobre dor (VAS - Visual Analogue Scale) de grupos de pacientes classificados como depressivos ou ansiosos (barras cheias) foram comparadas com as pontuações de grupos sem depressão ou ansiedade (barras vazias). Foram utilizados os questionários BDI (Beck Depression Inventory) e BAl (Beck Anxiety Inventory) para classificar os pacientes dentre os grupos de depressão e ansiedade, respectivamente. ${ }^{*} p<0,05$.

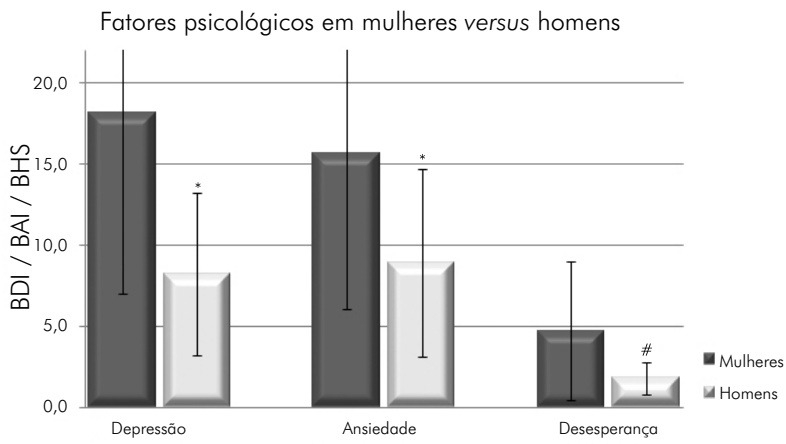

Figura 5

Prevalência de depressão, ansiedade e desesperança. As pontuações do questionário sobre depressão (BDI - Beck Depression Inventory), ansiedade (BAI - Beck Anxiety Inventory) e desesperança (BHS - Beck Hopelessnes Scale) foram analisados separadamente entre grupos de mulheres (barras cheias) e de homens (barras vazias).

${ }^{*} p<0,003$ e $\# p<0,02$. 
depressão, a ansiedade e a desesperança apareceram com maior intensidade nas mulheres (Figura 4). No total, 69\% de todos os casos de depressão e $57 \%$ de todos os casos de ansiedade eram mulheres (dados não mostrados).

Posteriormente, fazendo uma correlação desses dados clínicos com o estado psicológico dos pacientes, foi possível destacar a intensificação dos sintomas de dor e desabilidade física quando o paciente está ansioso em comparação a pacientes não-ansiosos (Figura 5). Existe a mesma tendência com pacientes em estado de depressão, apesar de não ter sido demonstrada estatisticamente devido ao número relativamente reduzido de dados.

\section{DISCUSSÃO}

Segundo estudo na população em geral, a prevalência, durante um ano, de distúrbios de ansiedade é de $10 \%{ }^{17}$, diferindo da prevalência de $56 \%$ que encontramos em pacientes com dor lombar crônica neste trabalho (Tabela 2). O mesmo acontece com os níveis de depressão, que estão em torno de $7 \%{ }^{18,19}$ na população em geral, tendo sido revelada uma prevalência global de $52 \%$ neste estudo (Tabela 2). Esses dados confirmam que existe uma relação substancial de ansiedade e depressão com quadros de dor lombar crônica.

Os quadros de depressão foram estratificados em prevalências de $3-5 \%$ para homens e $8-10 \%$ para mulheres na população em geral ${ }^{18,19} \mathrm{e}$, neste trabalho, observaram-se prevalências de $64 \%$ em mulheres e $28 \%$ em homens (dados não mostrados). É possível afirmar que a depressão aliada à dor crônica de coluna acomete um número maior de pessoas do sexo feminino, porém o aumento normalizado (mulhermulher e homem-homem) em relação à depressão na população em geral é o mesmo, ou seja, sete vezes maior.

Várias pesquisas mostram que diversas alterações psicológicas pioram o quadro de dor e restrição física do paciente, mostrando que pacientes com depressão apresentam predomínio de pensamentos negativos ${ }^{20}$, baixo limiar de dor ${ }^{21}$ e maiores restrições funcionais ${ }^{22}$. Além de modificar parâmetros somáticos, quadros emocionais nocivos dificultam ou mesmo impedem a reabilitação depois de diferentes tratamentos da coluna e podem ser preditivos de piores resultados de evolução clínica $^{23-27}$. Estes e outros estudos comprovaram que, pela identificação pré-operatória de alguns aspectos psicológicos (como depressão, ansiedade, hostilidade e desesperança), os pacientes portadores desses fatores evoluem de forma pior em relação aos outros pacientes, alertando quanto à necessidade de abordagens desta questão.

Outros parâmetros psicológicos, como a escala de psicastenia $^{28}$ e as escalas de histeria e hipocondria ${ }^{29-31}$, também comprovaram a influência negativa de fatores emocionais em resultados após cirurgias de coluna.

Porém, existem processos emocionais que ajudam na recuperação, como a boa capacidade de enfrentamento, ou seja, pensamentos, sentimentos e comportamentos específicos do indivíduo para lidar com a dor de forma adaptativa $^{32}$, mostrando resultados animadores para pacientes com dores de coluna que utilizam essas ferramentas aliadas à esperança e ao afastamento de pensamentos catastróficos $^{33,34}$. Pelo que pudemos observar no presente trabalho, há uma alta prevalência de desesperança entre os pacientes com dor lombar crônica, fato que pode vir a atrapalhar os resultados clínicos no período pós-cirúrgico.

Além de fatores internos, algumas interferências externas também foram comprovadamente influenciadoras de resultados clínicos. Mostramos uma prevalência de 43,8\% de afastamento do trabalho, corroborando dados que mostram que a situação de afastamento e compensação do trabalhador é muito prevalente e de extrema importância em pacientes que sofrem de problemas na coluna ${ }^{35}$. É comprovado que estes pacientes tendem a apresentar piores prognósticos ${ }^{36-38}$, fato que novamente evidencia a importância da identificação pré-operatória desses complicadores. Em outros trabalhos, são referidos ainda maiores graus de dor e piores resultados quando existe a presença de incentivos fiscais $^{10}$, reforço por parte da família ${ }^{38,39}$, insatisfação ou processos para afastamento do trabalho ${ }^{40,41}$, solicitação de pensão $0^{42} \mathrm{e}$ insatisfação quanto ao empregador ${ }^{43}$.

\section{CONCLUSÃO}

Como estabelecido anteriormente, a dor e seu alívio são parâmetros subjetivos, cada pessoa possui uma relação diferente com eles, assim, as variáveis emocionais e sociais influeciam diretamente nessa dinâmica, que não é determinada somente por terapias de intervenção somática.

A grande maioria dos pacientes que sofrem de dor lombar crônica apresenta problemas emocionais como depressão, ansiedade e desesperança, além de estar sujeita a fatores socioeconômicos determinantes. É necessária a identificação desses componentes antes de um procedimento terapêutico, para que haja uma abordagem mais completa e eficaz.

\section{REFERÊNCIAS}

1. World Health Organization. Chronic diseases and health promotion. Chronic rheumatic conditions. Geneva: WHO; 2010. [cited 2010 Jun 8]. Available from: http://www.who.int/chp/topics/ rheumatic/en/index.html
2. Scientific approach to the assessment and management of activity-related spinal disorders. A monograph for clinicians. Report of the Quebec Task Force on Spinal Disorders. Spine (Phila Pa 1976). 1987;12(7 Suppl):S1-59.
3. Ebmeier KP, Donaghey C, Steele JD. Recent developments and current controversies in depression. Lancet. 2006;367(9505):153-67. Comment in: Lancet. 2006;367(9518):1235. Lancet. 2006;367(9505):86. Lancet. 2006;367(9518):1235-6. Lancet. 2006;367(9518):1236. Lancet. 2006;367(9518):1236-7. 
4. Katon W, Ciechanowski P. Impact of major depression on chronic medical illness. J Psychosom Res. 2002;53(4):859-63.

5. Kim TS, Pae CU, Hong CK, Kim JJ, Lee CU, Lee SJ, et al. Interrelationships among pain, disability, and psychological factors in young Korean conscripts with lumbar disc herniation. Mil Med. 2006;171(11):1113-6.

6. Turk DC. Biopsychosocial perspective on chronic pain. In: Gatchel RJ, Turk DC, editors. Psychological approaches to pain management. New York: Guilford Press; 1996. p. 3-32.

7. Schulberg HC, Katon WJ, Simon GE, Rush AJ. Best clinical practice: guidelines for managing major depression in primary medical care. $\mathrm{J}$ Clin Psychiatry. 1999;60 Suppl 7:1926; discussion 27-8.

8. Murray CJL, Lopez AD, editors. The global burden of disease: a comprehensive assessment of mortality and disability from diseases, injuries, and risk factors in 1990 and projected to 2020. Boston: Harvard School of Public Health/World Health Organization/World Bank; 1996.

9. Arnow BA, Hunkeler EM, Blasey CM, Lee J, Constantino MJ, Fireman $\mathrm{B}$, et al. Comorbid depression, chronic pain, and disability in primary care. Psychosom Med. 2006;68(2):262-8.

10.Chapman RC. Pain: the perception of noxious events. In: Sternbach RA, editor. The psychology of pain. New York: Raven Press; 1978. p. 169-202.

11. Beck AT, Ward CH, Mendelson M, Mock J, Erbaugh J. An inventory for measuring depression. Arch Gen Psychiatry. 1961;4:561-71.

12.Beck AT, Steer RA, Carbin MG. Psychometric properties of the Beck Depression Inventory: Twenty-five years of evaluation. Clin Psychol Rev. 1988;8(1):77-100.

13.Beck AT, Epstein N, Brown G, Steer RA. An inventory for measuring clinical anxiety: psychometric properties. J Consult Clin Psychol. 1988;56(6):893-7.

14.Beck AT, Steer RA. BHS Beck hopelessness scale. New York: The Psychological Corporation; 1988.

15.Downie WW, Leatham PA, Rhind VM, Wright V, Branco JA, Anderson JA. Studies with pain rating scales. Ann Rheum Dis. 1978;37(4):378-81.
16. Roland M, Fairbank J. The RolandMorris Disability Questionnaire and the Oswestry Disability Questionnaire. Spine (Phila Pa 1976). 2000;25(24):3115-24. Review. Erratum in: Spine 2001;26(7):847.

17. Somers JM, Goldner EM, Waraich P, Hsu L. Prevalence and incidence studies of anxiety disorders: a systematic review of the literature. Can J Psychiatry. 2006;51(2):100-13. Comment in: Evid Based Ment Health. 2006;9(4):115.

18. Kessler RC, Berglund P, Demler O, Jin R, Merikangas KR, Walters EE. Lifetime prevalence and age-of-onset distributions of DSM-IV disorders in the National Comorbidity Survey Replication. Arch Gen Psychiatry. 2005;62(6):593-602. Erratum in: Arch Gen Psychiatry. 2005;62(7):768. Merikangas, Kathleen R [added]. Comment in: Arch Gen Psychiatry. 2005;62(6):590-2. CNS Drugs. 2005;19(8):717-8.

19.Murphy JM, Laird NM, Monson RR, Sobol AM, Leighton AH. A 40-year perspective on the prevalence of depression: the Stirling County Study. Arch Gen Psychiatry. 2000;57(3):20915. Comment in: Arch Gen Psychiatry. 2000;57(3):223-4. Arch Gen Psychiatry. 2000;57(3):227-8

20. Seligman M. Learned helplessness and depression in animals and men. In: Spence JT, Carson R, Thibaut J, editors. Behavioral approaches to therapy. Morristown, NJ: General Learning Press; 1976. p. 111-26.

21.Merskey H. The effect of chronic pain upon the response to noxious stimuli by psychiatric patients. J Psychosom Res. 1965;8:405-19.

22.Kremer EF, Block AR, Atkinson JJ. Assessment of pain behavior: factors that distort self-report. In: Melzack $\mathrm{R}$, editor. Pain measurement: an assessment. New York: Raven Press; 1983. p. 165-71

23. Rush AJ, Polatin P, Gatchel RJ. Depression and chronic low back pain: establishing priorities in treatment. Spine (Phila Pa 1976). 2000;25(20):2566-71.

24.Keefe FJ, Rumble ME, Scipio CD, Giordano LA, Perri LM. Psychological aspects of persistent pain: current state of the science. J Pain. 2004;5(4):195211.
25.Mannion AF, Junge A, Taimela S, Müntener M, Lorenzo K, Dvorak J. Active therapy for chronic low back pain: part 3. Factors influencing self-rated disability and its change following therapy. Spine (Phila $\mathrm{Pa}$ 1976). 2001;26(8):920-9.

26.Kjelby-Wendt G, Styf JR, Carlsson SG. The predictive value of psychometric analysis in patients treated by extirpation of lumbar intervertebral disc herniation. J Spinal Disord. 1999;12(5):375-9.

27.Trief PM, Grant W, Fredrickson B. A prospective study of psychological predictors of lumbar surgery outcome. Spine (Phila Pa 1976). 2000;25(20):2616-21. Comment in: Spine (Phila Pa 1976). 2002;27(11):1230-1.

28. Turk DC, Rudy TE. Toward an empirically derived taxonomy of chronic pain patients: integration of psychological assessment data. J Consult Clin Psychol. 1988;56(2):233-8.

29. Cashion EL, Lynch WJ. Personality factors and results of lumbar disc surgery. Neurosurgery. 1979;4(2):141-5.

30.Long CJ. The relationship between surgical outcome and MMPI profiles in chronic pain patients. J Clin Psychol. 1981;37(4):744-9.

31.Herron L, Turner JA, Ersek M, Weiner P. Does the Millon Behavioral Health Inventory (MBHI) predict lumbar laminectomy outcome? A comparison with the Minnesota Multiphasic Personality Inventory (MMPI). J Spinal Disord. 1992;5(2):188-92.

32.Brown GK, Nicassio PM. Development of a questionnaire for the assessment of active and passive coping strategies in chronic pain patients. Pain. 1987;31(1):53-64.

33. Rosensteil AK, Keefe FJ. The use of coping strategies in chronic low back pain patients: relationship to patient characteristics and current adjustment. Pain. 1983;17(1):33-44.

34. Block AR, Kremer EF, Gaylor M. Behavioral treatment of chronic pain: the spouse as a discriminative cue for pain behavior. Pain. 1980;9(2):243-52.

35. Hee HT, Whitecloud TS 3rd, Myers L, Gaynor J, Roesch W, Ricciardi JE. SF-36 health status of workers compensation cases with spinal disorders. Spine J. 2001;1(3):176-82.

36. Haddad GH. Analysis of 2932 workers' compensation back injury cases. The impact on the cost to the system. Spine (Phila Pa 1976). 1987;12(8):765-9. 
37. Greenough CG, Fraser RD. The effects of compensation on recovery from low-back injury. Spine (Phila Pa 1976). 1989;14(9):947-55.

38. Schade V, Semmer N, Main CJ, Hora J, Boos N. The impact of clinical, morphological, psychosocial and work-related factors on the outcome of lumbar discectomy. Pain. 1999;80(12):239-49.

39. Block AR, Ohnmeiss DD, Guyer RD, Rashbaum RF, Hochschuler SH. The use of presurgical psychological screening to predict the outcome of spine surgery. Spine J. 2001;1(4):274-82.
40.Davis RA. A long-term outcome analysis of 984 surgically treated herniated lumbar discs. J Neurosurg. 1994;80(3):514-21.

41.Junge A, Dvorak J, Ahrens S. Predictors of bad and good outcomes of lumbar disc surgery. A prospective clinical study with recommendations for screening to avoid bad outcomes. Spine (Phila Pa 1976). 1995;20(4):460-8.

42. Mannion AF, Elfering A. Predictors of surgical outcome and their assessment. Eur Spine J. 2006;15 Suppl 1:S93-108.
43.DeGood DE, Kiernan B. Perception of fault in patients with chronic pain. Pain. 1996;64(1):153-9.

\section{Correspondência:}

Leonardo Oliveira

Rua Vergueiro, 1.421 - Vila Mariana

CEP 04101-000 - São Paulo (SP), Brasil Tel: (1 1 ) 2936-8838

E-mail: leonardo@luizpimenta.com.br 